\title{
Lie Group Analysis of Natural Convective Flow from a Convectively Heated Upward Facing Radiating Permeable Horizontal Plate in Porous Media Filled with Nanofluid
}

\author{
Md. Jashim Uddin, ${ }^{1}$ W. A. Khan, $^{2}$ and A. I. Md. Ismail ${ }^{1}$ \\ ${ }^{1}$ School of Mathematical Sciences, Universiti Sains Malaysia, 11800 Penang, Malaysia \\ ${ }^{2}$ Department of Engineering Sciences, Pakistan Navy Engineering College, \\ National University of Sciences and Technology, Karachi 75350, Pakistan
}

Correspondence should be addressed to Md. Jashim Uddin, jashim_74@yahoo.com

Received 9 February 2012; Revised 19 March 2012; Accepted 20 March 2012

Academic Editor: Srinivasan Natesan

Copyright (c) $2012 \mathrm{Md}$. Jashim Uddin et al. This is an open access article distributed under the Creative Commons Attribution License, which permits unrestricted use, distribution, and reproduction in any medium, provided the original work is properly cited.

Two-dimensional, steady, laminar and incompressible natural convective flow of a nanofluid over a connectively heated permeable upward facing radiating horizontal plate in porous medium is studied numerically. The present model incorporates Brownian motion and thermophoresis effects. The similarity transformations for the governing equations are developed by Lie group analysis. The transformed equations are solved numerically by Runge-Kutta-Fehlberg fourth-fifth order method with shooting technique. Effects of the governing parameters on the dimensionless velocity, temperature and nanoparticle volume fraction as well as on the dimensionless rate of heat and mass transfer are presented graphically and the results are compared with the published data for special cases. Good agreement is found between numerical results of the present paper and published results. It is found that Lewis number, Brownian motion and convective heat transfer parameters increase the heat and mass transfer rates whilst thermophoresis decreases both heat and mass transfer rates.

\section{Introduction}

Nanoparticles are made from various materials, such as oxide ceramics $\left(\mathrm{Al}_{2} \mathrm{O}_{3}, \mathrm{CuO}\right)$, nitride ceramics ( $\mathrm{AlN}, \mathrm{SiN})$, carbide ceramics $(\mathrm{SiC}, \mathrm{TiC})$, metals $(\mathrm{Cu}, \mathrm{Ag}, \mathrm{Au})$, semiconductors, $\left(\mathrm{TiO}_{2}, \mathrm{SiC}\right)$, carbon nanotubes, and composite materials such as alloyed nanoparticles $\mathrm{Al}_{70} \mathrm{Cu}_{30}$ or nanoparticle core-polymer shell composites. Nanofluids aim to achieve the maximum possible thermal properties at the minimum possible concentrations (preferably $<1 \%$ by volume) by uniform dispersion and stable suspension of nanoparticles 
(preferably $<10 \mathrm{~nm}$ ) in host fluids [1]. Present heat transfer industries require high performance heat transfer equipment. The idea of improving heat transfer performance of fluids with the inclusion of solid particles was first introduced by Maxwell [2]. But, suspensions involving milli or microsized particles create problems, such as fast sedimentation, clogging of channels, high pressure drop, and severe erosion of system boundaries. To overcome these difficulties Choi [3] used ultrafine nanoparticles with base fluid termed as nanofluid. Modern material technologies facilitated the manufacturing of nanometer-sizes particles. Nanofluids have superior thermophysical properties like high thermal conductivity, minimal clogging in flow passages, long-term stability and homogeneity. Nanofluids have several industrial applications such as in electronics, automotive, and nuclear applications where efficient heat dissipation is necessary. According to Schaefer [4], nanobiotechnology is a fast growing field of research and application in many domains such as in medicine, pharmacy, cosmetics, and agroindustry. Advances in nanoelectronics, nanophotonics, and nanomagnetics have seen the arrival of nanotechnology as a distinct discipline in its own right [5].

A good number of research papers have been published on nanofluids to understand their performance so that they can be used to enhance the heat transfer in various industrial applications. A review of convective transport in nanofluids was conducted by Buongiorno [6]. Khan and Aziz [7] studied natural convection flow of nanofluid past a vertical plate with uniform heat flux. The Cheng and Minkowycz [8] problem was investigated by Nield and Kuznetsov [9] for nanofluid where the model incorporates the effect of Brownian motion and thermophoresis. Kuznetsov and Nield [10] presented a similarity solution of natural convective boundary-layer flow of a nanofluid past a vertical plate. An analytical study on the onset of convection in a horizontal layer of a porous medium with the Brinkman model and the Darcymodel filled with a nanofluid was presented by Kuznetsov and Nield [11, 12]. Godson et al. [13] presented the recent experimental and theoretical studies on convective heat transfer in nanofluids, their thermophysical properties, and applications and clarifies the challenges and opportunities for future research. Vajravelu et al. [14] studied convective heat transfer in the flow of viscous $\mathrm{Ag}$ water and $\mathrm{Cu}$ water nanofluids over a stretching surface. Noghrehabadi et al. [15] studied effect of partial slip boundary condition on the flow and heat transfer of nanofluids past stretching sheet prescribed constant wall temperature. Very recently, Aziz and Khan [16] studied similarity analysis of natural convective flow of a nanofluid over a convectively heated vertical plate.

According to previous researchers, for example, Aboeldahab and Azzam [17] radiation must be considered in calculating thermal effects in many new engineering processes occurring at high temperatures, such as the nuclear reactor cooling system, gas turbines, the various propulsion devices for aircraft, missiles, satellites, and space vehicles and various devices for space technology, underground nuclear wastes disposal, and so forth. Due to diverse applications of radiation, many investigators investigate the effect of radiation on the hydrodynamic or hydromagnetic or hydroelectric boundary layer flow over different geometries under different boundary conditions. A few examples are the papers by Cortell [18], Bataller [19], and Ishak et al. [20]. Gbadeyan et al. [21] present a numerical analysis of boundary layer flow of a nanofluid due over a linearly stretching sheet in the presence of thermal radiation. Very recently, Chamkha et al. [22] investigated mixed convective boundary-layer flow over an isothermal radiating vertical wedge placed in a porous medium filled with a nanofluid numerically using Keller box method.

Fluid flow and heat transfer in porous media have many engineering applications such as postaccidental heat removal in nuclear reactors, solar collectors, drying processes, storage of radioactive nuclear waste, heat exchangers, geothermal energy recovery and crude 
oil extraction, ground water pollution, thermal energy storage, building construction and flow through filtering media, separation processes in chemical industries [23]. Reviews of the fundamental theories and experiments of thermal convection in porous media with practical applications are presented in the books by Nield and Bejan [24], Vadasz [25], Vafai [26]. The classical problem of free convective flow in a porous medium near a horizontal flat plate was first investigated by Cheng and Chang [27]. After his pioneering works several authors such as Chang and Cheng [28], Shiunlin and Gebhart [29], Merkin and Zhang [30] and Chaudhary et al. [31] have extended the problem in various aspects. Gorla and Chamkha [32] presented a boundary layer analysis for the free convection flow of nanofluid over a horizontal upward facing plate in a porous medium numerically. Khan and Pop [23] extended this problem for nanofluid. Above investigators considered isothermal or isoflux thermal boundary conditions. However, the idea of using the thermal convective heating boundary condition was introduced by Aziz [33] to analyze Blasius flow. Following him, several authors, for example, Yao et al. [34], Uddin et al. [35], Magyari [36], and Yacob et al. [37] among others, used this boundary condition to study convective phenomena.

Above investigators found similarity solutions via dimensional analysis which can find only one particular type of similarity independent variable of the form $\eta=c y x^{r}$, where $r$ is a numerical constant and $c$ is a dimensional constant [38]. However, if one deals with the governing partial differential equations by Lie group analysis, then one can obtain former similarity transformation as well as some new forms $[39,40]$. Sometime it is extremely difficult to transform the PDEs to ODEs by using dimensional analysis. On the other hand, reduction of PDEs with boundary conditions to ODEs is much easier by use of Lie group analysis. The number of independent variables of PDEs can be reduced by one if the PDEs remain invariant under Lie group of transformations and the new system contains one less independent variable than the original one. This methodology can be applied $(n-1)$ times to reduce a boundary value problem of PDEs having $n$ number of independent variables to a boundary value problem of ODEs. The solution of reduced equations is much easier than the solutions of the original system of PDEs [41]. Hence, Lie group of transformations may be considered as the generalization of dimensional analysis. It is successfully applied in many areas such as in mathematical physics, applied and theoretical mechanics and applied mathematics and in the transport phenomena [42, 43]. Avramenko et al. [44] presented that the symmetrical properties of the turbulent boundary-layer flows and other turbulent flows are studied utilizing the Lie group theory technique.

The aim of the present study is to investigate the effect of thermophoresis, the Brownian motion, radiation and the thermal convective boundary condition on the boundary layer flow of a nanofluid over an upward facing radiating permeable horizontal plate numerically. A possible application of this problem is in the design of furnace where the transfer of heat from surfaces occurs simultaneously by radiation and convection. Also, the interaction of solar radiation with the earth's surface fabricates complex free convection patterns and hence complicates the studies associated with the weather forecasting and marine environment for predicting free convection patterns in oceans and lakes. Using similarity transformations developed by Lie group analysis, the governing partial differential equations are reduced to a set of coupled nonlinear ordinary differential equations with the corresponding boundary conditions. The effect of emerging flow controlling parameters on the dimensionless axial velocity, the temperature, the nanoparticle volume fraction, the rate of heat transfer, and the rate of nanoparticle volume fraction is investigated and shown graphically and discussed. 


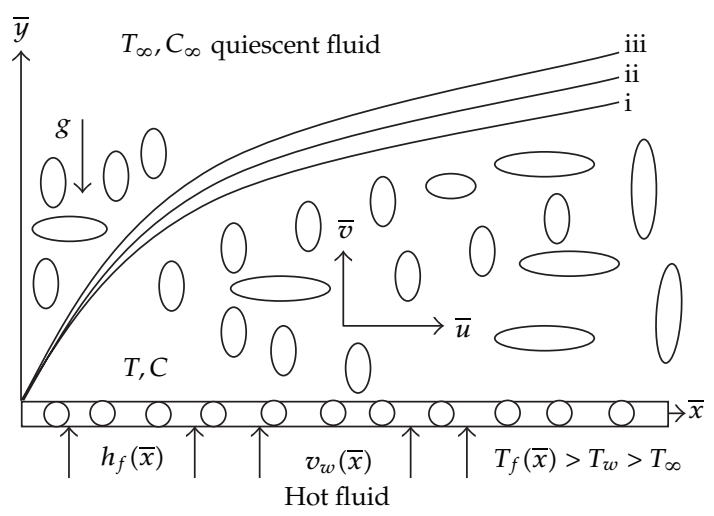

(i) Nanoparticle volume fraction boundary layer

(ii) Thermal boundary layer

(iii) Momentum boundary layer

Figure 1: Coordinate system and flow model.

\section{Formulation of the Problem}

We consider a two-dimensional $(\bar{x}, \bar{y})$ laminar free convective boundary layer flow past a permeable upward facing horizontal plate with radiation effects in a porous media filled with a nanofluid (Figure 1). The temperature $T$ and the nanoparticle volume fraction $C$ take constant values $T_{w}$ and $C_{w}$ at the boundary whilst $T_{\infty}$ and $C_{\infty}$ at free stream. Bottom of the plate is heated by convection from a hot fluid at temperature $T_{f}$ which gives a variable heat transfer coefficient $h_{f}(\bar{x})$. It is assumed that $T_{f}>T_{w}>T_{\infty}$. The Oberbeck-Boussinesq approximation is employed. The following four field equations represent the conservation of mass, momentum, thermal energy, and nanoparticles, respectively. The field variables are $\vec{V}$ : Darcy velocity vector, $T$ : the temperature, and $C$ : the nanoparticle volume fraction [23]:

$$
\begin{gathered}
\nabla \cdot \vec{V}=0, \\
\frac{\rho_{f}}{\varepsilon} \frac{\partial \vec{V}}{\partial t}=-\nabla P-\frac{\mu}{K} \vec{V}+\left[C \rho_{P}+(1-C)\left\{\rho_{f}\left(1-\beta\left(T-T_{\infty}\right)\right)\right\}\right] \vec{g}, \\
\left(\rho C_{P}\right)_{f}\left(\frac{\partial T}{\partial t}+\vec{V} \cdot \nabla T\right)=k_{m} \nabla^{2} T+\varepsilon\left(\rho C_{P}\right)_{P}\left[D_{B} \nabla C \cdot \nabla T+\left(\frac{D_{T}}{T_{\infty}}\right) \nabla T \cdot \nabla T\right]+\frac{16 \sigma_{1} T_{\infty}^{3}}{3 \kappa_{1}} \frac{\partial^{2} T}{\partial \bar{y}^{2}} \\
\frac{\partial C}{\partial t}+\vec{V} \cdot \nabla C=D_{B} \nabla^{2} C+\left(\frac{D_{T}}{T_{\infty}}\right) \nabla^{2} T .
\end{gathered}
$$

We write $\vec{V}=(\bar{u}, \bar{v})$. 
Here $\rho_{f}$ is the density of the base fluid, $\mu$ is the dynamic viscosity of the base fluid, $\beta$ is the volumetric expansion coefficient of nanofluid, $\rho_{p}$ is the density of the nanoparticles, $\left(\rho C_{P}\right)_{f}$ is the heat effective heat capacity of the fluid, $\left(\rho C_{P}\right)_{P}$ is the effective heat capacity of the nanoparticle material, $k_{m}$ is effective thermal conductivity of the porous medium, $\varepsilon$ is the porosity, $K$ is permeability of the porous media, $\vec{g}$ is the gravitational acceleration, $\sigma_{1}$ is the Sefan-Boltzman constant, and $k_{1}$ is the Rosseland mean absorption coefficient. Here $D_{B}$ stands for the Brownian diffusion coefficient and $D_{T}$ stands for the thermophoretic diffusion coefficient. To ignore an advective term and a Forchheimer quadratic drag term in the momentum equation, we assumed that the flow is slow.

Consider a steady state flow. In keeping with the Oberbeck-Boussinesq approximation and an assumption that the nanoparticle concentration is dilute, and with a suitable choice for the reference pressure, we can linearize the momentum equation and write (2.2) as

$$
0=-\nabla P-\frac{\mu}{K} \vec{V}+\left[\left(\rho_{P}-\rho_{f \infty}\right)\left(C-C_{\infty}\right)+\left(1-C_{\infty}\right) \rho_{f \infty} \beta\left(T-T_{\infty}\right)\right] \vec{g} .
$$

Making the standard boundary layer approximation based on an order of magnitude analysis to neglect the small order terms, we have the governing equations

$$
\begin{gathered}
\frac{\partial \bar{u}}{\partial \bar{x}}+\frac{\partial \bar{v}}{\partial \bar{y}}=0, \\
\frac{\partial P}{\partial \bar{x}}=-\frac{\mu}{K} \bar{u}, \\
\frac{\partial P}{\partial \bar{y}}=-\frac{\mu}{K} \bar{v}+\left[\left(1-C_{\infty}\right) \rho_{f \infty} g \beta\left(T-T_{\infty}\right)-\left(\rho_{P}-\rho_{f \infty}\right) g\left(C-C_{\infty}\right)\right], \\
\bar{u} \frac{\partial T}{\partial \bar{x}}+\bar{v} \frac{\partial T}{\partial \bar{y}}=\alpha_{m} \frac{\partial^{2} T}{\partial \bar{y}^{2}}+\tau\left[D_{B} \frac{\partial C}{\partial \bar{y}} \frac{\partial T}{\partial \bar{y}}+\left(\frac{D_{T}}{T_{\infty}}\right)\left(\frac{\partial T}{\partial \bar{y}}\right)^{2}\right]+\frac{16 \sigma_{1} T_{\infty}^{3}}{3\left(\rho c_{p}\right)_{f} \mathcal{K}_{1}} \frac{\partial^{2} T}{\partial \bar{y}^{2}}, \\
\bar{u} \frac{\partial C}{\partial \bar{x}}+\bar{v} \frac{\partial C}{\partial \bar{y}}=D_{B} \frac{\partial^{2} C}{\partial \bar{y}^{2}}+\left(\frac{D_{T}}{T_{\infty}}\right) \frac{\partial^{2} T}{\partial \bar{y}^{2}},
\end{gathered}
$$

where $\alpha_{m}=k_{m} /\left(\rho c_{P}\right)_{f}$ is the thermal diffusivity of the fluid and $\tau=\varepsilon\left(\rho C_{P}\right)_{p} /\left(\rho C_{P}\right)_{f}$ is a parameter.

The boundary conditions are taken to be [35]

$$
\begin{gathered}
\bar{v}=-v_{w}(\bar{x}), \quad-k \frac{\partial T}{\partial \bar{y}}=h_{f}(\bar{x})\left(T_{f}-T_{w}\right), \quad C=C_{w}, \quad \text { at } \bar{y}=0, \\
\bar{u} \longrightarrow 0, \quad T \longrightarrow T_{\infty}, \quad C \longrightarrow C_{\infty} \quad \text { as } \bar{y} \longrightarrow \infty
\end{gathered}
$$


Here $v_{w}(\bar{x})$ : mass transfer velocity. The following nondimensional variables are introduced to make (2.6)-(2.11) dimensionless

$$
\begin{aligned}
& x=\frac{\bar{x}}{L \sqrt{R a}}, \quad y=\frac{\bar{y}}{L}, \quad u=\frac{\bar{u} L}{\alpha_{m} \sqrt{R a}}, \quad v=\frac{\bar{v} L}{\alpha_{m}}, \quad \theta=\frac{T-T_{\infty}}{\Delta T}, \quad \phi=\frac{C-C_{\infty}}{\Delta C}, \\
& \Delta T=T_{f}-T_{\infty}, \quad \Delta C=C_{w}-C_{\infty},
\end{aligned}
$$

where $L$ is the plate characteristic length and $R a=g K \beta\left(1-C_{\infty}\right) \Delta T L /\left(\alpha_{m} v\right)$ is the Rayleigh number. A stream function $\psi$ defined by

$$
u=\frac{\partial \psi}{\partial y}, \quad v=-\frac{\partial \psi}{\partial x}
$$

is introduced into (2.6)-(2.11) to reduce number of dependent variables and equations. Note that (2.6) is satisfied identically. We are then left with the following three dimensionless equations:

$$
\begin{gathered}
\frac{\partial^{2} \psi}{\partial y^{2}}+\frac{\partial \theta}{\partial x}-N r \frac{\partial \phi}{\partial x}=0 \\
\frac{\partial \psi}{\partial y} \frac{\partial \theta}{\partial x}-\frac{\partial \psi}{\partial x} \frac{\partial \theta}{\partial y}-\frac{\partial^{2} \theta}{\partial y^{2}}-N b \frac{\partial \theta}{\partial y} \frac{\partial \phi}{\partial y}-N t\left(\frac{\partial \theta}{\partial y}\right)^{2}-R \frac{\partial^{2} \theta}{\partial y^{2}}=0 \\
L e\left[\frac{\partial \psi}{\partial y} \frac{\partial \phi}{\partial x}-\frac{\partial \psi}{\partial x} \frac{\partial \phi}{\partial y}\right]-\frac{\partial^{2} \phi}{\partial y^{2}}-\frac{N t}{N b} \frac{\partial^{2} \theta}{\partial y^{2}}=0
\end{gathered}
$$

The boundary conditions in (2.11) become

$$
\begin{gathered}
\frac{\partial \psi}{\partial x}=-\frac{L v_{w}(x)}{\alpha_{m}}, \quad \frac{\partial \theta}{\partial y}=-\frac{h_{f} L}{k}(1-\theta), \quad \phi=1 \quad \text { at } y=0 \\
\frac{\partial \psi}{\partial y} \longrightarrow 0, \quad \theta \longrightarrow 0, \quad \phi \longrightarrow 0 \quad \text { as } y \longrightarrow \infty
\end{gathered}
$$

Five parameters in (2.14) are $N t, N b, N r, R$, and $L e$ and they stand for the thermophoresis parameter, the Brownian motion parameter, the buoyancy ratio parameter, radiation parameter, and the Lewis number, respectively, which are defined by

$$
\begin{aligned}
& N t=\tau D_{T} \Delta T / \alpha_{m} T_{\infty}, \quad N b=\tau D_{B} \Delta C / \alpha_{m}, \quad L e=\alpha_{m} / D_{B}, \\
& N r=\left(\rho_{P}-\rho_{f \infty}\right) \Delta C / \rho_{f \infty} \beta\left(1-C_{\infty}\right) \Delta T, \quad R=\frac{16 \sigma_{1} T_{\infty}^{3}}{3\left(\rho c_{p}\right)_{f} k_{1} \alpha_{m}} .
\end{aligned}
$$




\section{Symmetries of the Problem}

By applying Lie group method to (2.14), the infinitesimal generator for the problem can be written as

$$
X=\xi_{1} \frac{\partial}{\partial x}+\xi_{2} \frac{\partial}{\partial y}+\tau_{1} \frac{\partial}{\partial \psi}+\tau_{2} \frac{\partial}{\partial \theta}+\tau_{3} \frac{\partial}{\partial \phi}
$$

where the coordinates $(x, y, \psi, \theta, \phi)$ transformed into the coordinates $\left(x^{*}, y^{*}, \psi^{*}, \theta^{*}, \phi^{*}\right)$. The infinitesimals $\xi_{1}, \xi_{2}, \tau_{1}, \tau_{2}$, and $\tau_{3}$ satisfies the following first order linear differential equations

$$
\begin{aligned}
\frac{d x^{*}}{d \varepsilon} & =\xi_{1}\left(x^{*}, y^{*}, \psi^{*}, \theta^{*}, \phi^{*}\right), & \frac{d y^{*}}{d \varepsilon}=\xi_{2}\left(x^{*}, y^{*}, \psi^{*}, \theta^{*}, \phi^{*}\right), \\
\frac{d \psi^{*}}{d \varepsilon} & =\tau_{1}\left(x^{*}, y^{*}, \psi^{*}, \theta^{*}, \phi^{*}\right), & \frac{d \theta^{*}}{d \varepsilon}=\tau_{2}\left(x^{*}, y^{*}, \psi^{*}, \theta^{*}, \phi^{*}\right), \\
\frac{d \theta^{*}}{d \varepsilon} & =\tau_{3}\left(x^{*}, y^{*}, \psi^{*}, \theta^{*}, \phi^{*}\right) . &
\end{aligned}
$$

Using commercial software Maple 13, it was found that the forms of the infinitesimals are

$$
\xi_{1}=c_{1} x+c_{2}, \quad \xi_{2}=\frac{2}{3} c_{1} y+c_{3}, \quad \tau_{1}=\frac{1}{3} c_{1} \psi+c_{6}, \quad \tau_{2}=c_{4} \theta, \quad \tau_{3}=c_{5} \phi,
$$

where $c_{i}(i=1,2,3,4,5,6)$ are arbitrary constants. Hence, the equations admit six finite parameters Lie group transformations. It is apparent that the parameters $c_{2}, c_{3}$, and $\mathrm{c}_{6}$ correspond to the translation in the variables $x, y$, and $\psi$, respectively. It is also observed that the parameters $c_{1}, c_{4}$, and $c_{5}$ correspond to the scaling in the variables $x, y, \psi, \theta$, and $\phi$, respectively. The generators corresponding to the infinitesimal given by (3.3) are as follows:

$$
X_{1}=x \frac{\partial}{\partial x}+\frac{2}{3} y \frac{\partial}{\partial y}+\frac{1}{3} \psi \frac{\partial}{\partial \psi}, \quad X_{2}=\frac{\partial}{\partial x}, \quad X_{3}=y \frac{\partial}{\partial y}, \quad X_{4}=\theta \frac{\partial}{\partial \theta}, \quad X_{5}=\frac{\partial}{\partial \phi} .
$$

We consider scaling transformations and hence set $c_{2}=c_{3}=c_{6}=0$.

Thus the infinitesimals become

$$
\xi_{1}=c_{1} x_{2}, \quad \xi_{2}=\frac{2}{3} c_{1} y, \quad \tau_{1}=\frac{1}{3} c_{1} \psi, \quad \tau_{2}=c_{4} \theta, \quad \tau_{3}=c_{5} \phi .
$$

In terms of differentials, we have

$$
\frac{d x}{c_{1} x}=\frac{d y}{(2 / 3) c_{1} y}=\frac{d \psi}{(1 / 3) c_{1} \psi}=\frac{d \theta}{c_{4} \theta}=\frac{d \phi}{c_{5} \phi} .
$$

Here $c_{1} \neq 0$. 


\subsection{Similarity Transformations}

From (3.6), $d x / c_{1} x=d y /(2 / 3) c_{1} y$, which on integration

$$
\frac{y}{x^{2 / 3}}=\text { constant }=\eta(\text { say })
$$

Similarly, $d x / c_{1} x=d \psi /(1 / 3) c_{1} \psi$ leads to

$$
\frac{\psi}{x^{1 / 3}}=\text { constant }=f(\eta)(\text { say }), \text { that is, } \psi=x^{1 / 3} f(\eta),
$$

where $f$ is arbitrary function of $\eta$.

Equations $d x / c_{1} x=d \theta / c_{4} \theta$ and $d x / c_{1} x=d \phi / c_{5} \phi$ lead to

$$
\theta=x^{c_{4} / c_{1}} \theta(\eta), \quad \phi=x^{c_{5} / c_{1}} \phi(\eta) .
$$

Thus from (3.7a)-(3.7c) we obtain the following similarity transformations:

$$
\eta=\frac{y}{x^{2 / 3}}, \quad \psi=x^{1 / 3} f(\eta), \quad \theta=x^{c_{4} / c_{1}} \theta(\eta), \quad \phi=x^{c_{5} / c_{1}} \phi(\eta) .
$$

Now, to make sure that $\theta \rightarrow 0, \phi \rightarrow 0$ as $\eta \rightarrow \infty$, set $c_{4}=c_{5}=0$.

Hence the similarity transformations are

$$
\eta=\frac{y}{x^{2 / 3}}, \quad \psi=x^{1 / 3} f(\eta), \quad \theta=\theta(\eta), \quad \phi=\phi(\eta)
$$

Thus the velocity component $u, v$ can be expressed as

$$
u=\frac{f^{\prime}}{x^{1 / 3}}, \quad v=-\frac{1}{3 x^{2 / 3}}\left(f-2 \eta f^{\prime}\right)
$$

where primes indicate differentiation with respect to similarity independent variable $\eta$. It is worth citing that the similarity transformations in (3.9) are consistent with the well-known similarity transformations reported in the paper of Cheng and Chang [27] for $\lambda=0$ in their paper, which support the validity of our analysis. 
Table 1: Comparison of present results with Gorla and Chamkha [32] for different values of buoyancy and nanofluid parameters.

\begin{tabular}{lcccc}
\hline & \multicolumn{2}{c}{ Present results } & \multicolumn{2}{c}{ Gorla and Chamkha [32] } \\
& $-\theta^{\prime}(0)$ & $-\phi^{\prime}(0)$ & $-\theta^{\prime}(0)$ & $-\phi^{\prime}(0)$ \\
\hline$N r$ & & $N b=0.3, N t=0.1, L e=10, r=1000, f_{w}=0$ & \\
\hline 0.1 & 0.32578 & 1.48242 & $3.26 E-01$ & 1.484164 \\
0.2 & 0.32385 & 1.46704 & $3.25 E-01$ & 1.468161 \\
0.3 & 0.32188 & 1.45125 & $3.22 E-01$ & 1.452664 \\
0.4 & 0.31985 & 1.43503 & $3.21 E-01$ & 1.436392 \\
0.5 & 0.31777 & 1.41833 & $3.19 E-01$ & 1.419499 \\
\hline$N t$ & \multicolumn{5}{c}{$N b=0.3, N r=0.5, L e=10, r=1000, f_{w}=0$} \\
\hline 0.1 & 0.31777 & 1.41833 & $3.19 E-01$ & 1.419499 \\
0.2 & 0.30486 & 1.41491 & $3.05 E-01$ & 1.416536 \\
0.3 & 0.2927 & 1.41561 & $2.93 E-01$ & 1.416866 \\
0.4 & 0.28125 & 1.41991 & $2.82 E-01$ & 1.421582 \\
0.5 & 0.27046 & 1.42737 & $2.71 E-01$ & 1.429226 \\
\hline$N b$ & & $N t=0.1, N r=0.5, L e=10, r=1000, f_{w}=0$ & \\
\hline 0.1 & 0.3672 & 1.32611 & $3.68 E-01$ & 1.327454 \\
0.2 & 0.34271 & 1.39216 & $3.43 E-01$ & 1.393615 \\
0.3 & 0.31777 & 1.41833 & $3.19 E-01$ & 1.419499 \\
0.4 & 0.29399 & 1.43428 & $2.94 E-01$ & 1.435464 \\
0.5 & 0.27161 & 1.44598 & $2.72 E-01$ & 1.44772 \\
\hline
\end{tabular}

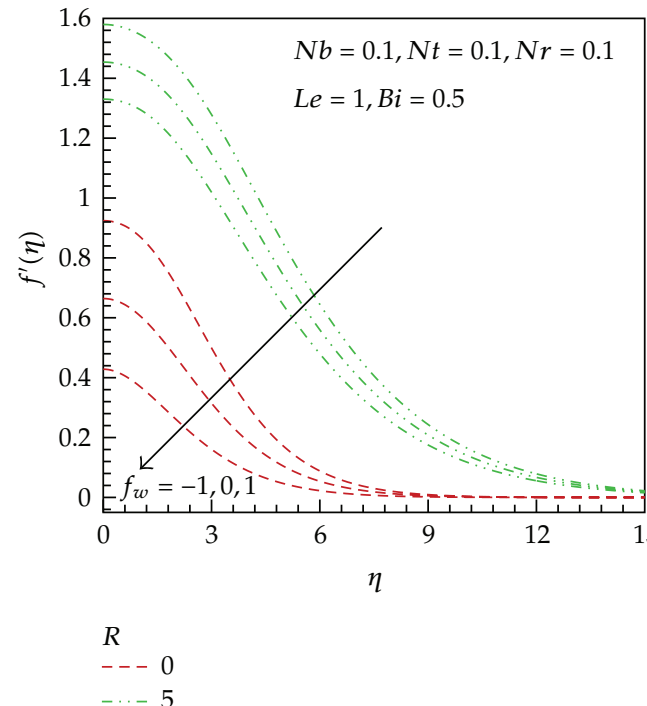

(a)

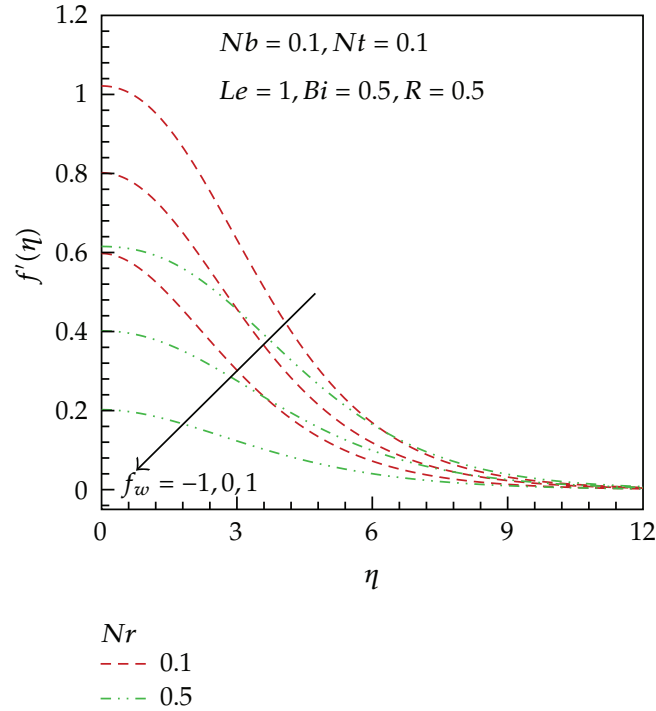

(b)

Figure 2: Effect of (a) radiation parameter, (b) buoyancy ratio parameter on the dimensionless velocity for different values of suction/injection parameter. 


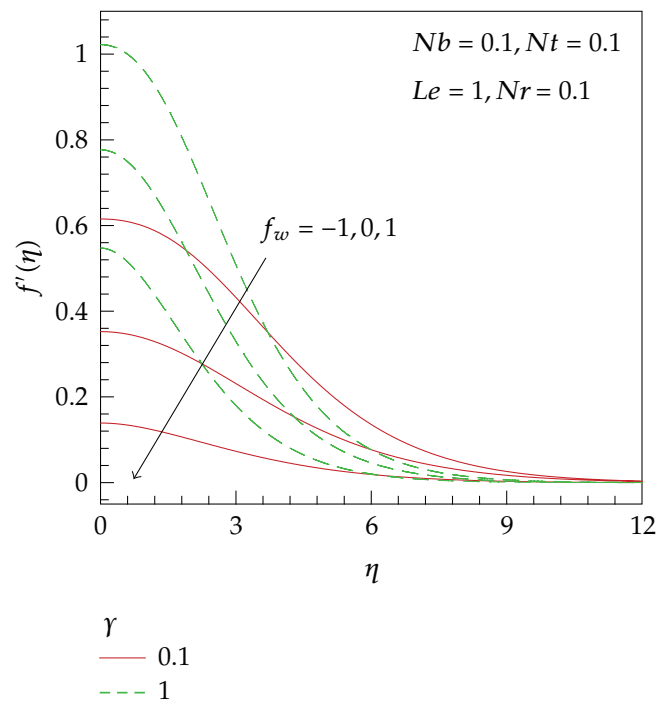

(a)

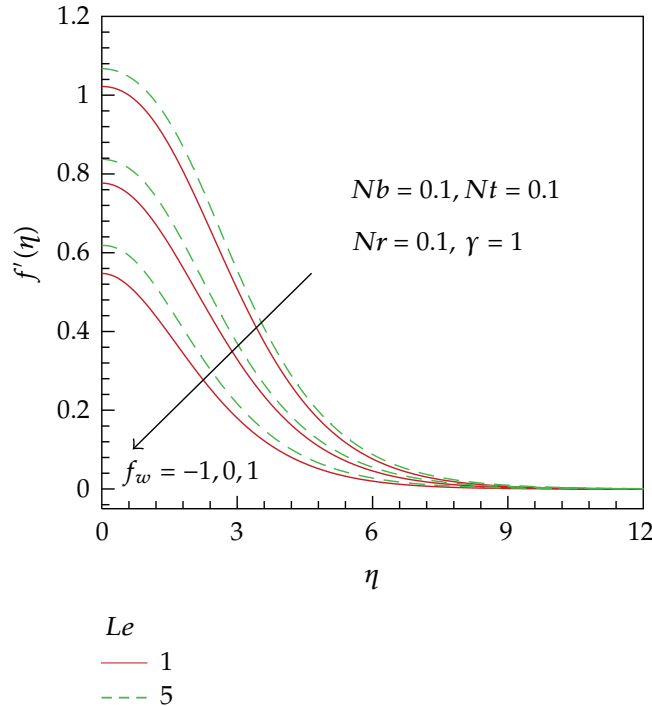

(b)

Figure 3: Effect of (a) Biot number, (b) Lewis number on the dimensionless velocity for different values of suction/injection parameter.

\subsection{Governing Similarity Equations}

Substituting the transformations in (3.9) into the governing (2.14) leads to the following nonlinear system of ordinary differential equations:

$$
\begin{gathered}
f^{\prime \prime}-\frac{2}{3} \eta\left(\theta^{\prime}-N r \phi^{\prime}\right)=0 \\
(1+R) \theta^{\prime \prime}+\frac{1}{3} f \theta^{\prime}+N b \theta^{\prime} \phi^{\prime}+N t \theta^{\prime 2}=0 \\
\phi^{\prime \prime}+\frac{L e}{3} f \phi^{\prime}+\frac{N t}{N b} \theta^{\prime \prime}=0
\end{gathered}
$$

subject to the boundary conditions

$$
f(0)=f w, \quad \theta^{\prime}(0)=-B i[1-\theta(0)], \quad \phi(0)=1, \quad f^{\prime}(\infty)=\theta(\infty)=\phi(\infty)=0 .
$$

Here $\mathrm{Nb}=0$ means there is no thermal transport due to buoyancy effects created as a result of nanoparticle concentration gradients and $f w=L v_{w} / 3 \alpha_{m}, f w>0$ corresponds to suction and $f w<0$ corresponds to injection, $B i=L h_{f} / k$ is the Biot number. It is mentioned that, for a true similarity solution, we must have

$$
h_{f}=\left(h_{f}\right)_{0} x^{-2 / 3}, \quad v_{w}=\left(v_{w}\right)_{0} x^{-2 / 3},
$$

where $\left(h_{f}\right)_{0}$ and $\left(v_{w}\right)_{0}$ are constants. 


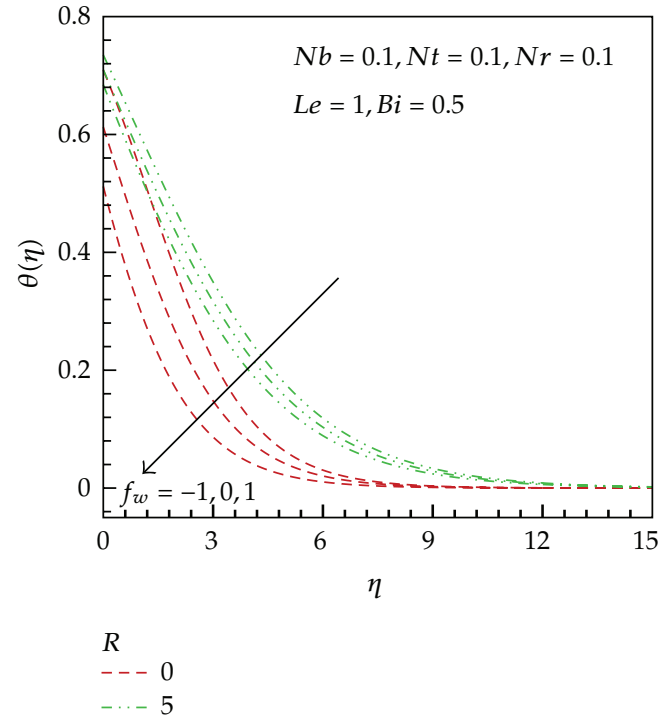

(a)

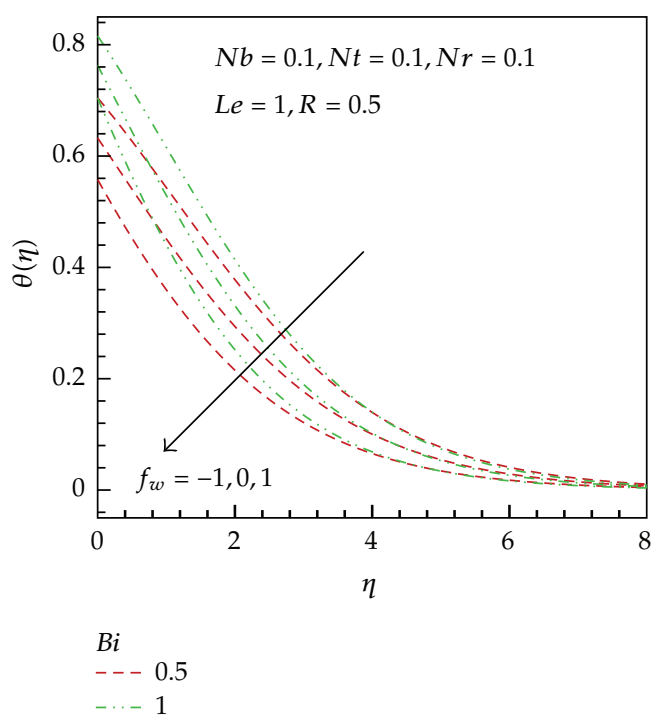

(b)

Figure 4: Effect of (a) radiation parameter, (b) Biot number on the dimensionless temperature for different values of suction/injection parameter.

\section{Comparisons with the Literature}

It is worth citing that in case of impermeable nonradiating plate $(f w=R=0)$ and for isothermal plate $(B i \rightarrow \infty)$, the problem under consideration reduces to the problem which has been recently investigated by Khan and Pop [23] and Gorla and Chamkha [32]. It is also worth mentioning that in case of impermeable non-radiating plate $(f w=R=0)$ and for constant wall temperature $(B i \rightarrow \infty)$, in the absence of buoyancy force $(N r=0)$, thermophoresis $(\mathrm{Nt}=0)$ and in the absence of Brownian motion $(\mathrm{Nb}=0)$, the problem under consideration reduces to the problem which was investigated by Cheng and Chang [27] for $\mathcal{\lambda}=0$ in their paper. It is further noted that in case of non-radiating plate $(R=0)$, the problem under consideration reduces to the problem which was recently investigated by Uddin et al. [35].

\section{Physical Quantities}

The parameters of engineering interest are the local skin friction factor $C_{f \bar{x}}$, the local Nusselt number $\mathrm{Nu}_{\bar{x}}$, and the local Sherwood number $\mathrm{Sh}_{\bar{x}}$, respectively. Physically, $C_{f \bar{x}}$ indicates wall shear stress, $\mathrm{Nu}_{\bar{x}}$ indicates the rate of heat transfer whilst $\mathrm{Sh}_{\bar{x}}$ indicates the rate of mass transfer. These quantities can be calculated from following relations:

$$
C_{f \bar{x}}=\frac{2 \mu}{\rho U_{r}^{2}}\left(\frac{\partial \bar{u}}{\partial \bar{y}}\right)_{\bar{y}=0}, \quad \mathrm{Nu}_{\bar{x}}=\frac{-\bar{x}}{T_{f}-T_{\infty}}\left(\frac{\partial T}{\partial \bar{y}}\right)_{\bar{y}=0}, \quad \operatorname{Sh}_{\bar{x}}=\frac{-\bar{x}}{C_{w}-C_{\infty}}\left(\frac{\partial C}{\partial \bar{y}}\right)_{\bar{y}=0} .
$$




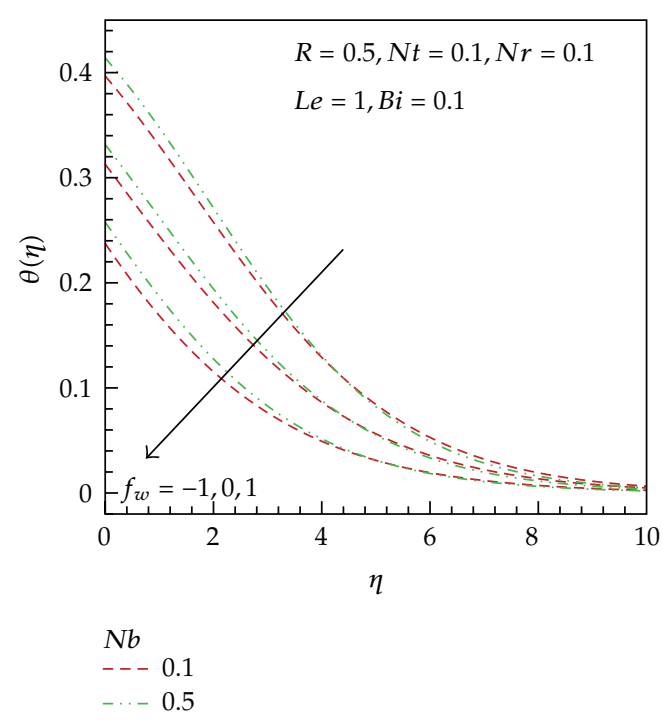

(a)

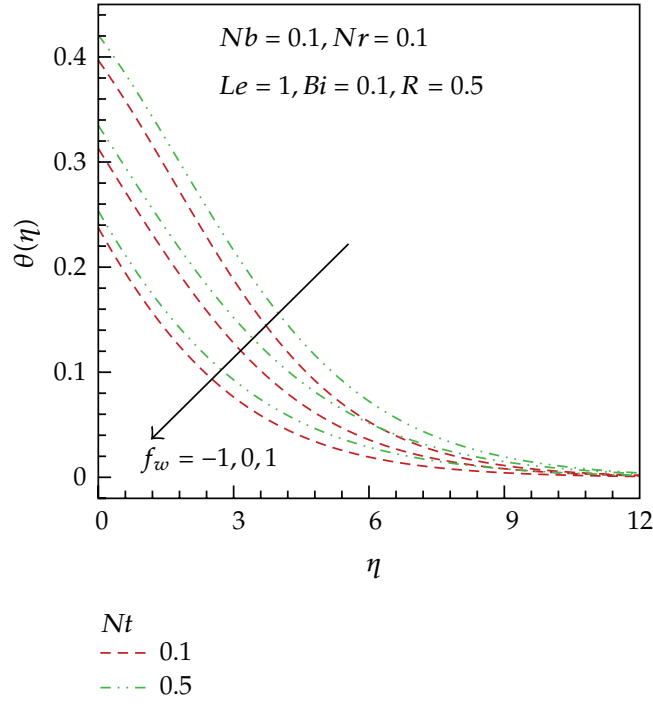

(b)

Figure 5: Effect of (a) Brownian motion parameter, (b) thermophoresis parameter on the dimensionless temperature for different values of suction/injection parameter.

By substituting from (2.12) and (3.9) into (5.1), it can be shown that physical quantities can be put in the following dimensionless form:

$$
\operatorname{Ra}_{\bar{x}} \operatorname{PrC}_{f \bar{x}}=2 f^{\prime \prime}(0), \quad \operatorname{Ra}_{\bar{x}}^{-1 / 3} \mathrm{Nu}_{\bar{x}}=-\theta^{\prime}(0), \quad \operatorname{Ra}_{\bar{x}}^{-1 / 3} \operatorname{Sh}_{\bar{x}}=-\phi^{\prime}(0) .
$$

Here $\operatorname{Ra}_{\bar{x}}=g K \beta\left(1-C_{\infty}\right) \Delta T \bar{x} /\left(\alpha_{m} v\right)$ is the local Rayleigh number, $\operatorname{Pr}=v / \alpha_{m}$ is the Prandt number for porous media, and $U_{r}=\left(1-C_{\infty}\right) g K \beta \Delta T / \alpha_{m}$ is reference velocity in porous media. Note that the local skin friction factor, the local Nusselt number, and the local Sherwood number are directly proportional to the numerical values of $f^{\prime \prime}(0),-\theta^{\prime}(0)$ and $-\phi^{\prime}(0)$, respectively.

\section{Results and Discussion}

The set of coupled nonlinear similarity Equations (3.11) with boundary conditions in (3.12) forms a two-point boundary value problem and has been solved numerically using an efficient Runge-Kutta-Fehlberg fourth-fifth order numerical method under Maple 14. To highlight the important features of the flow velocity, temperature, nanoparticle volume fraction, the heat transfer rate, and the nanoparticle volume fraction transfer rate, the obtained numerical results are displayed graphically. Numerical computations are done for $0 \leq R \leq 5,-1 \leq f_{w} \leq 1,0.1 \leq N b \leq 0.5,0.1 \leq N t \leq 0.5,0 \leq N r \leq 0.5$, $0 \leq B i \leq 5.0$ and $1 \leq L e \leq 5$. The results of the dimensionless heat transfer rates- $\theta^{\prime}(0)$ and the dimensionless nanoparticle volume fraction rate $-\phi^{\prime}(0)$ are compared with the most recent results reported by Gorla and Chamkha [32] for special case in Table 1 and found to be in excellent agreement with each of values of $N r, N b$, and $N t$. This supports the validity 


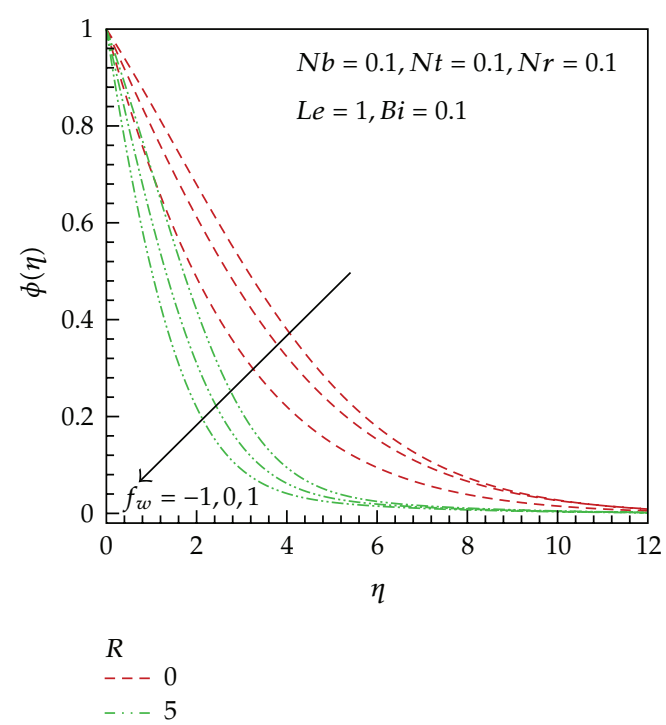

(a)

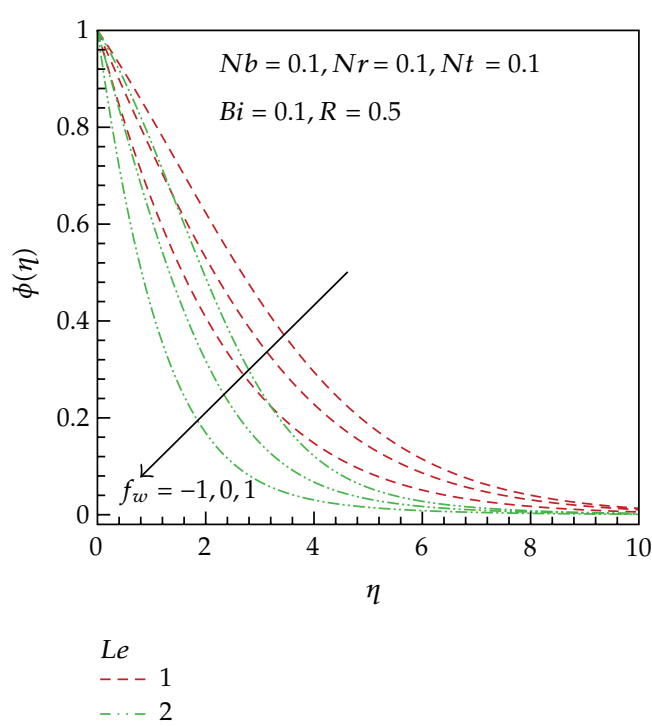

(b)

Figure 6: Effect of (a) radiation parameter, (b) Lewis number on the dimensionless nanoparticle volume fraction for different values of suction/injection parameter.

of our other graphical results for dimensionless velocity, temperature, nanoparticle volume fraction, heat transfer, and nanoparticle volume fraction transfer rates.

\subsection{Velocity Profiles}

Figures 2 and 3 exhibit the dimensionless axial velocity profiles for various values of the emerging flow controlling parameters. Dimensionless velocity and corresponding velocity boundary layer thickness are decreased with increasing values of the mass transfer velocity both for radiating $(R=5)$ and nonradiating $(R=0)$ plate. In Figure $2(a)$, it is found that dimensionless velocity increases with the increasing of the radiation parameter. It is apparent from Figure 2(b) that the dimensionless velocity decreases with rising of the buoyancy ratio parameter. The velocity is reduced with the suction; reverse phenomena are observed in case of the injection, as expected. The dimensionless velocity is elevated with rising of the Biot number and the Lewis number (Figure 3).

\subsection{Temperature Profiles}

Variation of the dimensionless temperature and corresponding thermal boundary layer thickness with radiation parameter, suction/injection parameter, the Biot number, thermophoresis, and Brownian motion parameters is shown in Figures 4 and 5, respectively.Temperature is increased with the increasing of radiation and Boit number (Figure 4). Physically, higher Biot number increases nanoparticle volume fraction as nanoparticle volume fraction distribution is driven by temperature distribution. The fluid on the right surface of the plate is heated up by the hot fluid on the left surface of the plate, making it lighter and flowing faster. 


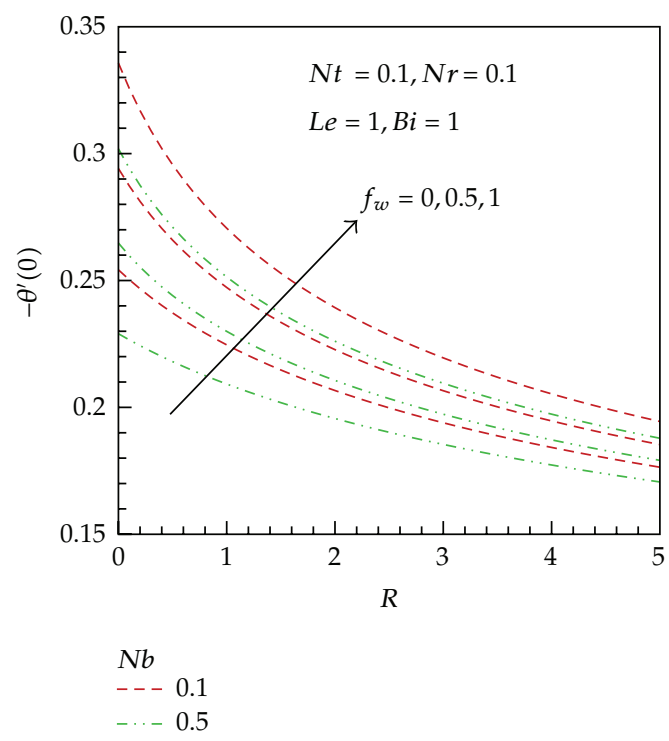

(a)

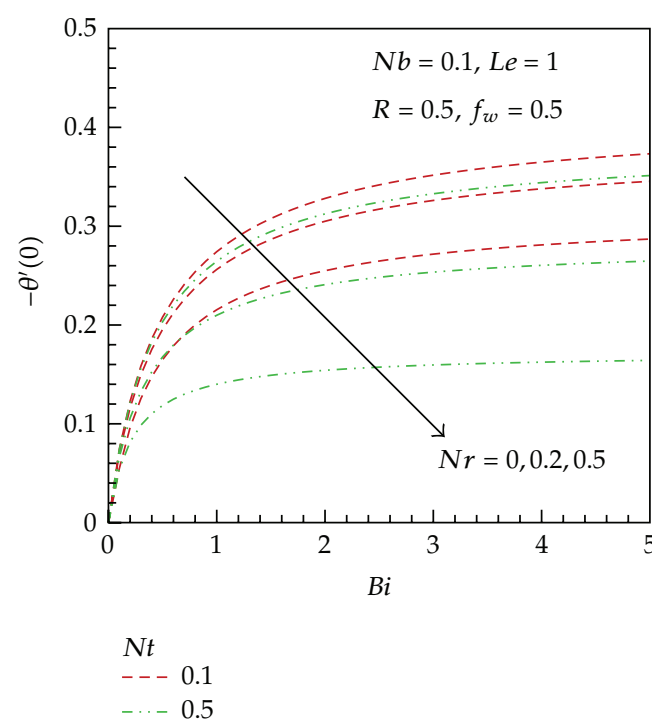

(b)

Figure 7: Variation of local heat transfer rate with (a) Brownian motion, suction/injection, and radiation parameters, (b) thermophoresis, buoyancy ratio, and Biot number.

Note that the temperature increases with the increasing of the Brownian motion and thermophoresis parameters when the plate is permeable or not (Figure 5). From Figures 4 and 5 , it is apparent that like regular fluid suction/injection parameter reduces the dimensionless temperature as expected.

\subsection{Nanoparticle Volume Fraction Profiles}

Figure 6 illustrates the impact of the controlling parameters on the dimensionless nanoparticle volume fraction inside the corresponding boundary layer. Dimensionless nanoparticle volume fraction is reduced due the enhance of both the radiation and the Lewis number when the plate is permeable or not (Figures 6(a) and 6(b)). Finally, from Figure 6, we found that the suction/injection parameter reduces the dimensionless nanoparticle volume fraction as in the case of regular fluid.

\subsection{Heat Transfer Rate}

The effect of various controlling parameters on the dimensionless heat transfer rate from a permeable horizontal upward facing plate with the thermal convective boundary condition in porous media is shown in Figure 7. From Figure 7(a), it is noticed that the dimensionless heat transfer rate decreases with an increase in thermophoresis and radiation parameter whilst it increases with the increasing of the suction velocity. It is further found from Figure 7(b) that the dimensionless heat transfer rate decreases with an increase in thermophoresis and buoyancy ratio parameter for permeable plate. We also noticed that heat transfer rate is 


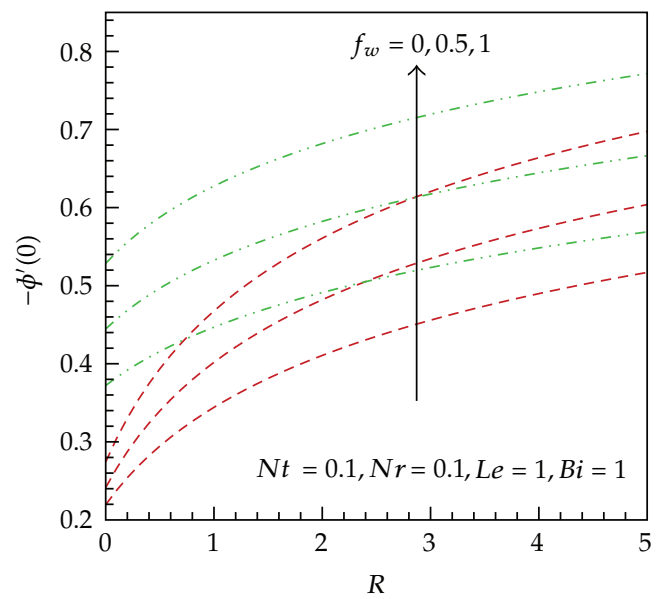

$$
\begin{array}{ll}
\mathrm{Nb} & \\
--- & 0.1 \\
-\cdot- & 0.5
\end{array}
$$

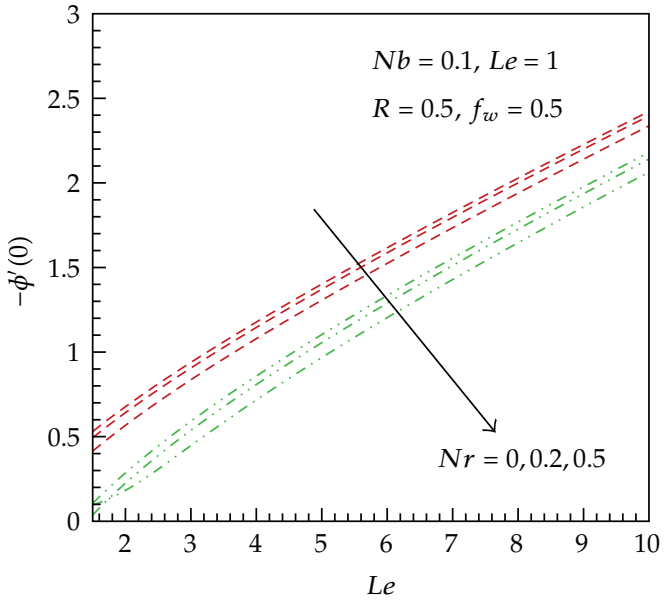

$N t$

$---0.1$

(a)

(b)

Figure 8: Variation of local mass transfer rate with (a) Brownian motion, radiation, and suction/injection parameters, (b) Lewis number, buoyancy ratio, and thermophoresis parameters.

a decreasing function of the radiation parameter (Figure $7(a)$ ) whilst it is increasing function of the Boit number (Figure 7(b)).

\subsection{Nanoparticle Volume Fraction Rate}

Figure 8 shows the effect of the radiation, the suction, thermophoresis, buoyancy ratio, and Lewis number parameters on the dimensionless nanoparticle volume fraction transfer rate from a permeable horizontal upward facing radiating plate in porous media. From Figure $8(a)$, we observed that the dimensionless nanoparticle volume fraction rate increases with an increase in Brownian motion, suction, and the radiation parameter. It is also found from Figure 8(b) that the dimensionless nanoparticle volume fraction rate decreases with an increase in both the thermophoresis and buoyancy ratio parameter for a permeable plate. It is further observed form Figure 8(b) that the Lewis number increases the dimensionless nanoparticle volume fraction rate, as in regular fluid.

\section{Conclusions}

We studied numerically a 2-D steady laminar viscous incompressible boundary layer flow of a nanofluid over an upward facing horizontal radiating permeable plate placed in the porous media considering the thermal convective boundary condition. The governing boundary layer equations are transformed into highly nonlinear coupled ordinary differential equations using similarity transformations developed by Lie group analysis, before being solved 
numerically. Following conclusions are drawn:

(i) the dimensionless velocity, the temperature, and the concentration decrease in case of the suction and increase in case of the injection; the phenomenon is reversed,

(ii) the Brownian motion, radiation, thermophoresis, and buoyancy ratio parameters decrease the heat transfer rate whilst the suction parameter and the Biot number enhance the heat transfer rate,

(iii) the radiation, Lewis number, Brownian motion, and the suction parameters cause to enhance nanoparticle volume fraction rate whilst thermophoresis and buoyancy ratio parameters lead to decreasing nanoparticle volume fraction rate.

\section{References}

[1] S. Kakaç, S. Özerinç, and A. G. Yazıcıŏlu, “Enhanced thermal conductivity of nanofluids: a state-ofthe-art review," Microfluid Nanofluid, vol. 8, pp. 145-170, 2010.

[2] J. C. Maxwell, A Treatise on Electricity and Magnetism, Clarendon Press, Oxford, UK, 2nd edition, 1981.

[3] S. U. S. Choi, "Enhancing thermal conductivity of fluids with nanoparticles," in Developments and Applications of Non-Newtonian Flows, D. A. Siginer and H. P. Wang, Eds., pp. 99-105, American Society of Mechanical Engineers, Baltimore, Md, USA, 1995.

[4] H. Schaefer, Nanoscience: The Science of the Small in Physics, Engineering. Chemistry, Biology and Medicine (Nanoscience and Technology), Springer, 2010.

[5] R. P. E. Sharma, Nanostructuring Operations in Nanoscale Science and Engineering, Kal.Mcgraw Hill, 2010.

[6] J. Buongiorno, “Convective transport in nanofluids," ASME Journal of Heat Transfer, vol. 128, pp. 240250, 2006.

[7] W. A. Khan and A. Aziz, "Natural convection flow of a nanofluid over a vertical plate with uniform surface heat flux," International Journal of Thermal Science, vol. 50, pp. 1207-1214, 2011.

[8] P. Cheng and W. J. Minkowycz, "Free convection about a vertical flat plate embedded in a porous medium with application to heat transfer from a dike," Journal of Geophysics Research, vol. 82, no. 14, pp. 2040-2044, 1977.

[9] D. A. Nield and A. V. Kuznetsov, "The Cheng-Minkowycz problem for natural convective boundarylayer flow in a porous medium saturated by a nanofluid," International Journal of Heat Mass Transfer, vol. 52, pp. 5792-5795, 2009.

[10] A. V. Kuznetsov and D. A. Nield, "Natural convective boundary-layer flow of a nanofluid past a vertical plate," International Journal of Thermal Science, vol. 49, pp. 243-247, 2010.

[11] A. V. Kuznetsov and D. A. Nield, "Thermal instability in a porous medium layer saturated by a nanofluid: brinkman model," Transport in Porous Media, vol. 81, no. 3, pp. 409-422, 2010.

[12] A. V. Kuznetsov and D. A. Nield, "Effect of local thermal non-equilibrium on the onset of convection in a porous medium layer saturated by a nanofluid," Transport in Porous Media, vol. 83, pp. 425-436, 2010.

[13] L. B. Godson, D. Raja, D. L. Mohan, and S. Wongwisesc, "Enhancement of heat transfer using nanofluids-an overview," Renewable and Sustainable Energy Reviews, vol. 14, pp. 629-641, 2010.

[14] K. Vajravelu, K. V. Prasad, J. Lee, C. Lee, I. Pop, and R. A. van Gorder, "Convective heat transfer in the flow of viscous Ag water and $\mathrm{Cu}$ water nanofluids over a stretching surface," International Journal of Thermal Sciences, vol. 50, pp. 843-851, 2011.

[15] A. Noghrehabadi, P. Rashid, and G. Mohammad, "Effect of partial slip boundary condition on the flow and heat transfer of nanofluids past stretching sheet prescribed constant wall temperature," International Journal of Thermal Sciences, vol. 54, pp. 253-261, 2012.

[16] A. Aziz and W. A. Khan, "Natural convective boundary layer flow of a nanofluid past a convectively heated vertical plate," International Journal of Thermal Science, vol. 52, pp. 83-90, 2012.

[17] E. M. Aboeldahab and G. E. A. Azzam, "Thermal radiation effects on MHD flow past a semi-infinite inclined plate in the presence of mass diffusion," Heat and Mass Transfer, vol. 41, no. 12, pp. 1056-1065, 2005. 
[18] R. Cortell, "Suction, viscous dissipation and thermal radiation effects on the flow and heat transfer of a power-law fluid past an infinite porous plate," Chemical Engineering Research and Design, vol. 89, pp. 85-93, 2011.

[19] R. C. Bataller, "Radiation effects in the Blasius flow," Applied Mathematics and Computation, vol. 198, no. 1, pp. 333-338, 2008.

[20] A. Ishak, N. A. Yacob, and N. Bachok, "Radiation effects on the thermal boundary layer flow over a moving plate with convective boundary condition," Meccanica, vol. 46, no. 4, pp. 795-801, 2011.

[21] J. A. Gbadeyan, M. A. Olanrewaju, and P. O. Olanrewaju, "Boundary layer flow of a nanofluid past a stretching sheet with a convective boundary condition in the presence of magnetic field and thermal radiation," Australian Journal of Basic and Applied Sciences, vol. 5, no. 9, pp. 1323-1334, 2011.

[22] A. J. Chamkha, S. Abbasbandy, A. M. Rashad, and K. Vajravelu, "Radiation effects on mixed convection over a wedge embedded in a porous medium filled with a nanofluid," Transport in Porous Media, vol. 91, pp. 261-279, 2012.

[23] W. A. Khan and I. Pop, "Free convection boundary layer flow past a horizontal flat plate embedded in a porous medium filled with a nanofluid," ASME Journal of Heat Transfer, vol. 133, no. 9, Article ID 094501, 4 pages, 2011.

[24] D. A. Nield and A. Bejan, Convection in Porous Media, Springer, New York, NY, USA, 3rd edition, 2006.

[25] P. Vadasz, Emerging Topics in Heat and Mass Transfer in Porous Media, Springer, New York, NY, USA, 2008.

[26] K. Vafai, Porous Media: Applications in Biological Systems and Biotechnology, CRC Press, New York, NY, USA, 2010.

[27] P. Cheng and I. D. Chang, "Buoyancy induced flows in a saturated porous medium adjacent to impermeable horizontal surfaces," International Journal of Heat Mass Transfer, vol. 19, pp. 1267-1272, 1976.

[28] I. D. Chang and P. Cheng, "Matched asymptotic expansions for free convection about an impermeable horizontal surface in a porous medium," International Journal of Heat Mass Transfer, vol. 26, pp. 163$173,1983$.

[29] D. S. Shiunlin and B. Gebhart, "Buoyancy-induced flow adjacent to a horizontal surface submerged in porous medium saturated with cold water," International Journal of Heat Mass Transfer, vol. 29, pp. 611-623, 1986.

[30] J. H. Merkin and G. Zhang, "On the similarity solutions for free convection in a saturated porous medium adjacent to impermeable horizontal surfaces," Wärme-und Stoffübertragung, vol. 25, pp. 179$184,1990$.

[31] M. A. Chaudhary, J. H. Merkin, and I. Pop, "Natural convection from a horizontal permeable surface in porous medium-numerical and asymptotic solutions," Transport in Porous Media, vol. 22, no. 3, pp. 327-344, 1996.

[32] R. S. R. Gorla and A. Chamkha, "Natural convective boundary layer flow over a horizontal plate embedded in a porous medium saturated with a nanofluid," Journal of Modern Physics, vol. 2, pp. 62-71, 2011.

[33] A. Aziz, "A similarity solution for laminar thermal boundary over a flat plate with a convective boundary condition," Communication in Nonlinear Science and Numerical Simulation, vol. 14, pp. 1064 1068, 2009.

[34] S. Yao, T. Fang, and Y. Zhong, "Heat transfer of a generalized stretching/shrinking wall problem with convective boundary conditions," Communication in Nonliear Science and Numerical Simulation, vol. 16, pp. 752-760, 2011.

[35] M. J. Uddin, W. A. Khan, and A. I. M. Ismail, "Free convection boundary layer flow from a heated upward facing horizontal flat plate embedded in a porous medium filled by a nanofluid and with a convective boundary condition," Transport in Porous Media, vol. 92, no. 3, pp. 867-881, 2012.

[36] E. Magyari, "Comment on "a similarity solution for laminar thermal boundary layer over a flat plate with a convective surface boundary condition" by A. Aziz, Comm.Non.Sci. Numer. Simul.' (2009).14, 1064'68," Communications in Nonlinear Science and Numerical Simulation, vol. 19, pp. 599-601, 2011.

[37] N. A. Yacob, A. Ishak, and I. Pop, "Falkner-Skan problem for a static or moving wedge in nanofluids," International Journal of Thermal Sciences, vol. 50, pp. 133-139, 2011.

[38] G. W. Bluman and S. Kumei, Symmetries and Differential Equations, vol. 81 of Applied Mathematical Sciences, Springer, New York, NY, USA, 1989.

[39] M. Jalil, S. Asghar, and M. Mushtaq, "Lie group analysis of mixed convection flow with mass transfer over a stretching surface with suction or injection," Mathematical Problems in Engineering, vol. 2010, Article ID 264901, 14 pages, 2010. 
[40] M. Pakdemirli and M. Yurusoy, "Similarity transformations for partial differential equations," SIAM Review, vol. 40, no. 1, pp. 96-101, 1998.

[41] G. W. Bluman and S. C. Anco, Symmetry and Integration Methods for Differential Equations, Springer, New York, NY, USA, 2009.

[42] P. J. Olver, Applications of Lie Groups to Differential Equations, vol. 107 of Graduate Texts in Mathematics, Springer, New York, NY, USA, 1986.

[43] H. Ibragimov and V. F. Kovalev, Approximate and Re-Norm Group symmetry, Springer, New York, NY, USA, 2009.

[44] A. A. Avramenko, S. G. Kobzar, I. V. Shevchuk, A. V. Kuznetsov, and L. T. Iwanisov, "Symmetry of turbulent boundary-layer flows: investigation of different eddy viscosity models," Acta Mechanica, vol. 151, pp. 1-14, 2001. 


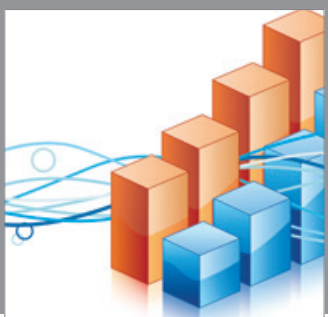

Advances in

Operations Research

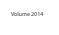

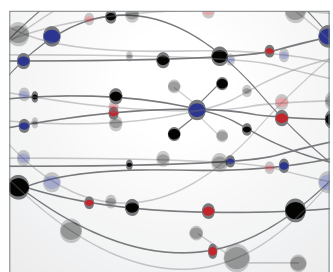

\section{The Scientific} World Journal
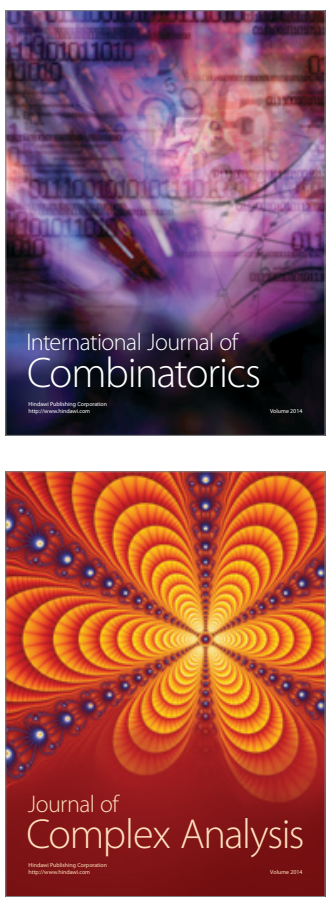

International Journal of

Mathematics and

Mathematical

Sciences
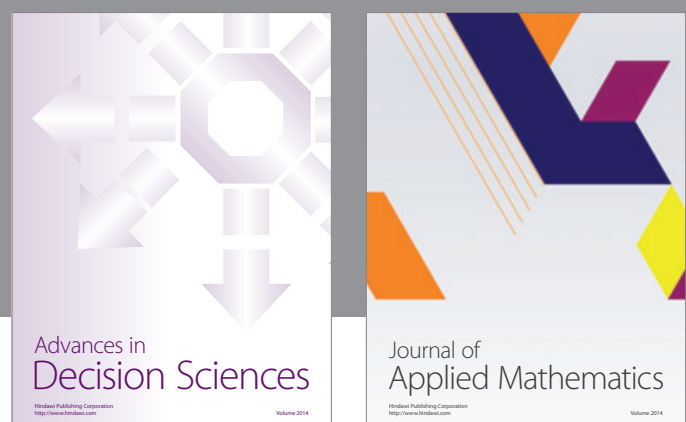

Journal of

Applied Mathematics
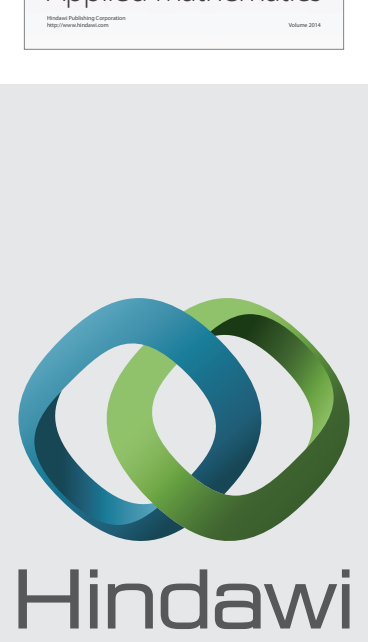

Submit your manuscripts at http://www.hindawi.com
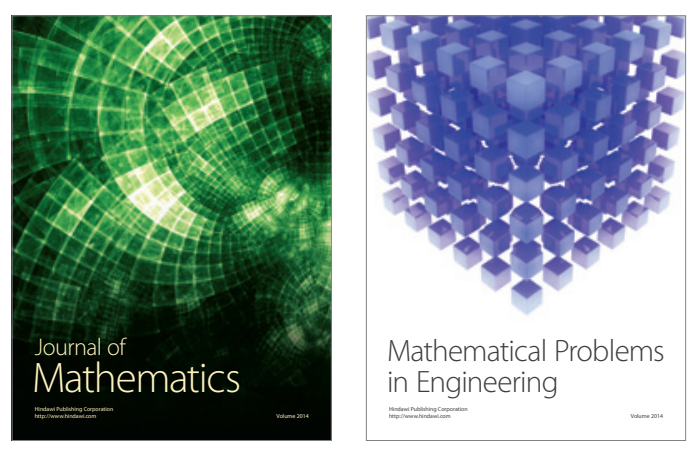

Mathematical Problems in Engineering
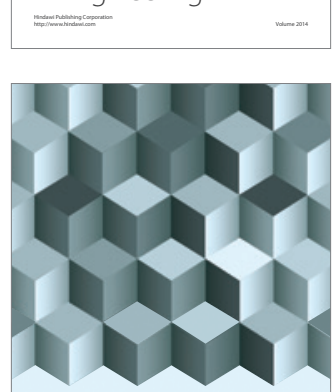

Journal of

Function Spaces
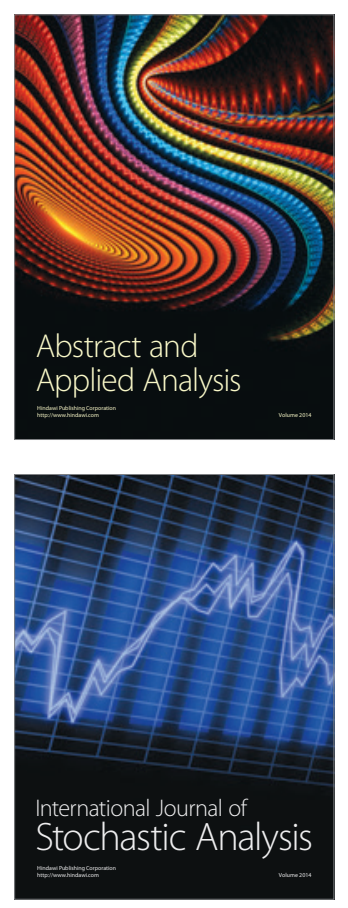

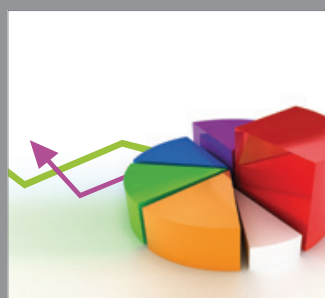

ournal of

Probability and Statistics

Promensencen
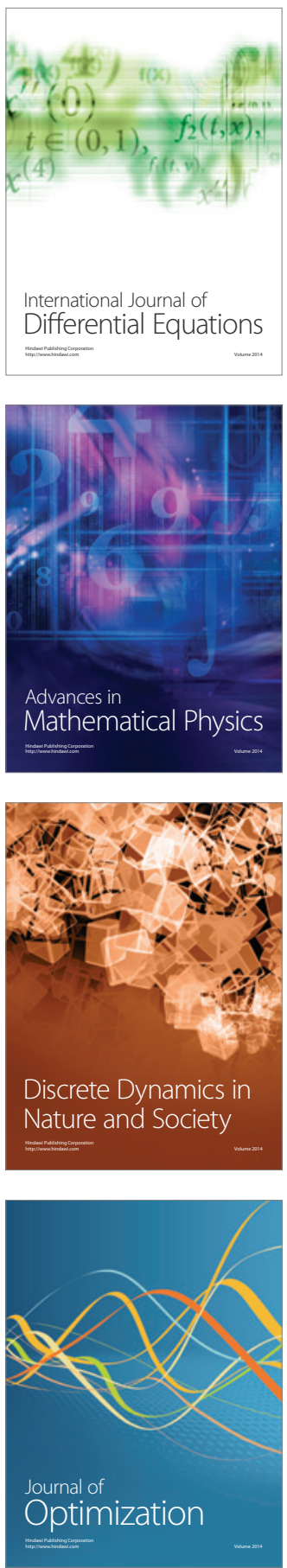